\section{A Tree-Based Forward Digest Protocol to Verify Data Integrity in Distributed Media Streaming}

\author{
Ahsan Habib, Member, IEEE, \\ Dongyan Xu, Member, IEEE, \\ Mikhail Atallah, Fellow, IEEE, \\ Bharat Bhargava, Fellow, IEEE, \\ and John Chuang, Member, IEEE
}

\begin{abstract}
We design a Tree-based Forward Digest Protocol (TFDP) to verify data integrity in distributed media streaming for content distribution. Several challenges arise, including the timing constraint of streaming sessions, the involvement of multiple senders, and the untrustworthiness of these senders. A comprehensive comparison is presented on the performance of existing protocols and TFDP, with respect to communication and computation overhead. Both simulation and Internet-based experimental results are presented to demonstrate the effectiveness of TFDP.
\end{abstract}

Index Terms-Data integrity, message digest, and media streaming.

\section{INTRODUCTION}

CONSIDER the following media data distribution scenario: A source content server called "Hollywood" first starts streaming a movie to its clients. When a sufficient number of clients have been served and have agreed to serve as redistributors, they will begin streaming the movie to other clients in the system. The distribution is supervised by the server: It authenticates requesting clients and gives them credentials to be served by the supplying clients, who will perform distributed media streaming only if proper credentials are presented. One key property of such distributed media streaming is that each streaming session involves multiple supplying clients due to the limited bandwidth contributed to the session by each of them. We note that, unlike traditional file sharing systems, a media streaming session allows continuous playback of media data during the session.

In such a "many-to-one" media streaming session, an untrustworthy supplying client (or "supplier") may corrupt any block of the media data. The verification of media data integrity thus becomes a critical task and poses a number of challenges. First, unlike authentication for multicast streaming [1], [11], [8], the suppliers cannot be assumed as trusted. In distributed media streaming, packets signed by one client may not be acceptable to other clients. Therefore, a client needs a point of reference to verify the media data it receives. Second, due to the real-time constraint of media streaming, data integrity verification needs to be performed in real-time. Third, the objective of verifying data integrity is not only to verify that the data are not corrupted, but also to validate that the media file is really what the client has requested. We note that other security problems exist in distributed media streaming, such as how to prevent the disclosure

- A. Habib and J. Chuang are with the School of Information Management and Systems, University of California at Berkeley, 102 South Hall, Berkeley, CA 94720. E-mail: \{habib,chuang\}@sims.berkeley.edu.

- D. Xu, M. Atallah, and B. Bhargava are with the Department of Computer Science, Purdue University, 250 N. University St., West Lafayette, IN 47907. E-mail: $\{d x u, m j a, b b\} @ c s . p u r d u e . e d u$.

Manuscript received 24 June 2004; revised 27 Oct. 2004; accepted 25 Jan. 2005; 18 May 2005.

For information on obtaining reprints of this article, please send e-mail to: tkde@computer.org, and reference IEEECS Log Number TKDE-0182-0604. of copyright-protected media data to unauthorized parties. These issues are outside the scope of this paper.

By presenting a comprehensive survey of existing protocols for data integrity verification, we show that they are either inapplicable or too expensive for distributed media streaming. We adopt the method of message digest and propose a Tree-based Forward Digest Protocol (TFDP). TFDP uses Merkle tree [7] and distributes data digest delivery overhead over the duration of a streaming session. The protocol works well with unreliable transport protocols. This is achieved by using Forward Error Correction (FEC) codes, especially for data digests. Our experiments show that TFDP is able to verify media data integrity with low communication and computation overhead.

TFDP is also applicable to Bittorrent-like file sharing applications that adopt the "multiple senders and single receiver" model similar to distributed media streaming. In addition, users are required to perform simultaneous uploads and downloads. By using TFDP, a receiver can verify data received from multiple suppliers block by block during a download session and, thus, it can become an uploading supplier at the same time.

The rest of the paper is organized as follows: Section 2 surveys related work. Section 3 presents TFDP. A comprehensive comparison among different solutions is presented in Section 4. Section 5 presents experimental results. Section 6 concludes this paper.

\section{Related Work}

To the best of our knowledge, there has been no prior study on data integrity verification for many-to-one distributed media streaming. In this section, we survey current related solutions and identify their limitations in supporting distributed media streaming.

Digital signature. A straightforward method to verify data integrity is to let the source server sign every packet (packet indicates the minimum unit of media transport, not an IP packet) or the hash of each packet with its private key using digital signature. A client can then verify the signed data using the server's public key. The RSA signature [14] verification has high computation overhead and is not suitable for real-time applications. Unlike RSA, one-time signature schemes such as [5], [9], [13] incur low verification overhead and latency. These schemes are usually used to sign multicast or broadcast streams. Rohatgi [15] proposed a $k$-time signature scheme which is more efficient than the one-time signature schemes. Still, the scheme generates 300 bytes for each signature.

Signature chain. Gennaro and Rohatgi [2] introduced techniques to sign offline and online digital streams. The first packet of an offline stream is signed and the hash of each packet is embedded in the next packet. The online scheme signs the initial packet and embeds the public key of a one-time signature in each packet, which is used to sign the subsequent packet. Although an elegant solution, it does not tolerate packet losses and it incurs high communication overhead.

Perrig et al. [10], [11] proposed TESLA and EMSS for efficient and secure multicast. TESLA embeds the signature of packet $p_{i}$ and the key to verify packet $p_{i-1}$ in packet $p_{i}$. The key of packet $p_{i}$ is sent in packet $p_{i+1}$. The adversary will see the key but it is too late to forge the signature. TESLA requires strict ordering of packets by the sender, which cannot be guaranteed in distributed many-to-one streaming. Furthermore, if the supplying clients generate keys and sign the digests as in TESLA, they may not be acceptable to other clients because clients are not assumed to be trustworthy.

Signature tree. Wong and Lam [16] studied data authenticity and integrity for lossy multicast streams. They proposed using Merkle signature tree to sign multicast streams. In their scheme, the root is signed to amortize one signature over multiple 
messages. Each packet contains the digests of all nodes necessary to compute the digest of the root and the signature of the root. As a result, the space requirement is rather high: 200 bytes in each packet using 1,024-bit RSA for a tree of 16 packets. The protocol we propose also uses Merkle tree. However, we significantly reduce the overhead by sending the digests of one subtree before sending any data. Thus, a packet does not need to carry all the digests that are required to verify its integrity.

Park et al. [8] proposed SAIDA that leverages erasure codes to amortize a single signature operation over multiple packets. In SAIDA, a block-a series of contiguous packets-of $a$ packets carries the encoded digests and signature of the block. The signature and digests are recoverable if the receiver gets any $b \leq a$ packets. This digest encoding is robust against bursty packet losses to a certain level. To reduce overhead, FEC is used to encode only digests, not data.

Both signature tree and SAIDA are designed for multicast where the sender signs packets and the receivers trust the sender. In our protocol, a receiver does not have to trust the suppliers. Unlike signature tree and SAIDA, we choose not to use digital signature to further reduce overhead.

Erasure codes and homomorphic hash function. Krohn et al. [4] use homomorphic hash function to verify erasure codes during a many-to-one bulk file transfer session. A client can verify each block on the fly while downloading a large file such as Linux ISO from multiple suppliers. Thus, the client does not have to wait until the end of the transfer to verify the entire file. While effective for file downloading, this verification scheme is not applicable to real-time media streaming because the client has to wait until the end of the file transfer to decode all blocks.

\section{Protocol Description}

In our protocol, we assume the existence of a trusted authority denoted as the Authentication Server (or "server") $S_{0}$. To request a distributed streaming session, a client will first authenticate itself with $S_{0}$ and obtain a point of reference to be used for data integrity verification during the streaming session. In our protocol, the reference data is only 20 bytes long for each requesting client. We first define the distributed media streaming model and then describe our protocol.

Streaming model. Client $P_{0}$ requests a media streaming session to be served by a set of supplying clients $\mathbb{P}=\left\{P_{1}, P_{2}, \ldots, P_{m}\right\}$. The set of suppliers is determined by a certain lookup and selection mechanism. The requested media file has a size of $F$ bytes and is divided into a set of $M$ blocks $\mathbb{B}=\left\{b_{1}, b_{2}, \ldots, b_{M}\right\}$. Each block consists of $l$ packets. We denote a block as $b_{i}=\left\{p_{i 1}, p_{i 2}, \ldots, p_{i l}\right\}$, where $p_{i j}$ is the $j$ th packet of the $i$ th block. Finally, a sequence of blocks is referred to as a group. During a streaming session, different suppliers transmit different packets of each block to the client, which will reconstruct the block. Details on packet assignment to suppliers can be found in [3].

Tree-based Forward Digest Protocol (TFDP). As in Tree Chaining proposed by Wong and Lam for multicast flows [16], we use Merkle tree to design TFDP. Merkle tree [7] generates onetime signature using one-way function tree and hash function. Each message to be signed corresponds to a node in the tree. Each node consists of verification parameters that are used to sign a message and to authenticate the verification parameters of subsequent nodes. The root of the tree is the public key for signature verification. We note that the idea of constructing oneway function tree has also been applied to key management in secure group communications [12], [6]. In fact, such tree construction is common in a number of application scenarios, but for different purposes: Rafaeli and Hutchison [12] as well as McGrew and Sherman [6] use it for key management, Merkle [7] uses it to

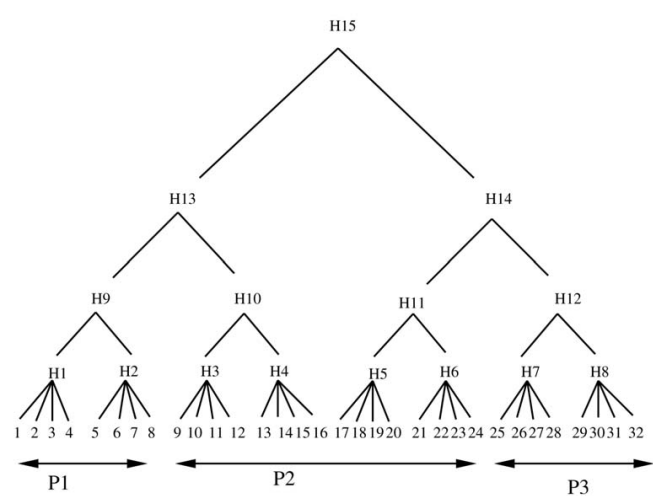

Fig. 1. A Merkle tree with 32 packets that belong to eight blocks. $P_{1}, P_{2}$, and $P_{3}$ are suppliers. $P_{1}$ will provide digests $H_{1}, H_{2}, H_{10}$, and $H_{14}$ that are required to verify the first two blocks. Similarly, $P_{2}$ will provide digests for the next four blocks and $P_{3}$ will provide digests for the last two blocks.

generate signatures, while we (TFDP) as well as Wong and Lam (Tree Chaining) use it to authenticate data streams.

In Tree Chaining, a file is divided into a number of blocks, with each block containing a set of packets. A signature tree is then constructed for each block with all packets of the block as leaves. The root of the tree is signed by a digital signature. In TFDP, however, we do not sign the root of every subtree that belongs to each block. Instead, we only compute digests to build a Merkle tree. The main difference between Tree Chaining and TFDP is that the former is designed for multicast flows (one-to-many) where each packet carries the necessary information for its authentication and thus incurs high overhead, while the latter is designed for many-to-one streaming where the overhead is amortized over a group of data blocks.

In TFDP, server $S_{0}$ generates the Merkle tree for a media file. The leaves of the tree are packets in the file. Each nonleaf node of the tree represents the digest of its children. The server enforces that a supplier keeps a minimum number of digests, so that the overhead of sending digests is amortized over a group of data blocks. During a streaming session, $N_{\min }$ digests are transmitted before transmitting the media data blocks. A higher $N_{\min }$ will reduce the block verification overhead. However, it will incur longer delay in the streaming session.

Fig. 1 shows a simple example with 32 packets that belong to eight blocks. Each nonleaf node $H_{i}$ represents the digest of its children. $P_{1}, P_{2}$, and $P_{3}$ are suppliers involved in the same streaming session. Let $P_{1}$ be assigned to provide digests for the first two blocks, $P_{2}$ for the next four blocks, and $P_{3}$ for the last two blocks. In this example, the digests provided by $P_{1}$ are $H_{1}, H_{2}$, $H_{10}$, and $H_{14} . P_{0}$ then computes $H_{9}$ from $H_{1}$ and $H_{2}, H_{13}$ from $H_{9}$ and $H_{10}$, and $H_{15}$ from $H_{13}$ and $H_{14}$, and compares $H_{15}$ against the digest supplied by server $S_{0}$. If there is a match, the belief in $H_{15}$ is transferred to all digests provided by $P_{1}$ because of the property of collision-free hash function. During the streaming session, media data sent by the three suppliers can be verified block by block using $H_{1}$ and $H_{2}$ provided by $P_{1} . P_{2}$ and $P_{3}$ operate in a similar fashion. We now describe the steps of TFDP:

- Step 1: Client $P_{0}$ authenticates itself with server $S_{0}$ by sending $E_{S_{0}}\left(D_{P_{0}}\left(M_{0}\right)\right)$, where the request message $M_{0}$ is signed by $P_{0}$ for nonrepudiation. Then, it is encrypted with the public key of $S_{0}$.

- $\quad$ Step 2: The server sends $E_{P_{0}}\left(D_{S_{0}}\left(H_{\text {root }}, T\right)\right)$ to $P_{0}$, where the digest of the root of the Merkle tree is $H_{\text {root }}$, which is encrypted with the public key of $P_{0}$ and signed by the private key of the server. $T$ is a timestamped ticket that 
TABLE 1

Comparison of Data Integrity Verification Protocols

\begin{tabular}{|c|c|c|c|c|c|c|c|c|}
\hline & $\begin{array}{l}\text { Allow } \\
\text { packet } \\
\text { loss }\end{array}$ & $\begin{array}{l}\text { Overhead: } \\
\text { server } \rightarrow P_{0} \\
\text { (Bytes) }\end{array}$ & $\begin{array}{l}\text { Overhead: } \\
\mathbb{P} \rightarrow P_{0} \\
\text { (Bytes) }\end{array}$ & $\begin{array}{l}\text { \# of hash } \\
\text { computation } \\
\text { by server }\end{array}$ & $\begin{array}{l}\text { \# of hash } \\
\text { computation } \\
\text { by } P_{0}\end{array}$ & $\begin{array}{l}\text { Sign by } \\
\text { server }\end{array}$ & $\begin{array}{l}\text { Verify } \\
\text { signature } \\
\text { by } P_{0}\end{array}$ & $\begin{array}{l}\text { FEC } \\
\text { decode } \\
\text { by } P_{0}\end{array}$ \\
\hline Tree Chaining & YES & 0 & $20 M l \log l+128 M l$ & $M(2 l-1)$ & $M(2 l-1)$ & $M$ & $M$ & - \\
\hline SAIDA & YES & 0 & $(20 l+128) M \alpha$ & $M(l+1)$ & $M(l+1)$ & $M$ & $M$ & $M$ \\
\hline TFDP & YES & 20 & $\begin{array}{l}20 M l \alpha+20[M+ \\
\left.\frac{M}{N_{\min }} \log \left(\frac{M}{N_{\min }}\right)\right] \\
\end{array}$ & $2 M-1$ & $\begin{array}{l}M l+M / N_{\min }\left[\left(N_{\min }\right.\right. \\
\left.-1)+\log \left(M / N_{\min }\right)\right]\end{array}$ & - & - & $M$ \\
\hline
\end{tabular}

$M$ is the total number of blocks of a file, $l$ is the size of one block in number of packets, and $\alpha$ is the FEC overhead factor.

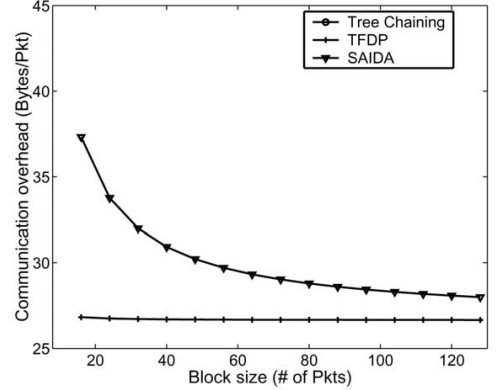

(a)

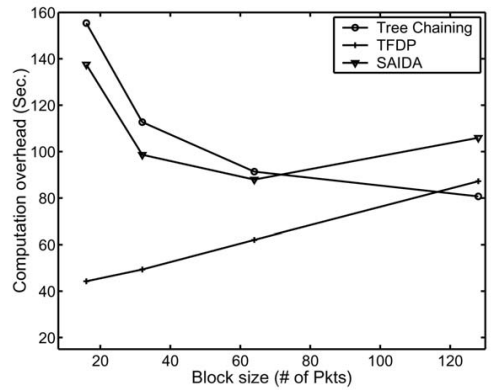

(b)

Fig. 2. Overhead of Tree Chaining, TFDP, and SAIDA for The Matrix movie (size: 1.3 GB). The communication overhead is per packet while the computation overhead is for the entire file. Tree Chaining has 208 bytes overhead per packet (not shown in the figure). (a) Communication overhead. (b) Computation overhead.

needs to be presented to each supplier to prevent a user from using the same ticket beyond a specific time period.

- Step 3: $P_{0}$ tells supplier $P_{i}$ in $\mathbb{P}$ to provide the digests needed to verify $N_{\min }$ blocks. If more than one supplier has all the digests, $P_{0}$ randomly selects one of them.

- $\quad$ Step 4: Supplier $P_{i}$ provides to $P_{0}$ all required digests to verify $N_{\min }$ data blocks.

- $\quad$ Step 5: If the root digest computed by $P_{0}$ matches the root digest obtained from the server, $P_{0}$ will trust the digests provided by $P_{i}$ and use them for data integrity verification during the streaming session. Otherwise, the protocol goes to Step 3 and obtains digests from a different supplier.

- $\quad$ Step 6: $P_{0}$ signals the suppliers in $\mathbb{P}$ to send media data.

- Step 7: The suppliers start the streaming session. Using the digests obtained in Step $4, P_{0}$ verifies every media data block reconstructed from packets transmitted by the suppliers. Once the streaming of $N_{\min }$ blocks is done, the protocol goes to Step 3 to repeat the process for the next $N_{\text {min }}$ blocks.

Fig. 1 is a binary tree if we exclude the leaves. Especially, a parent node of the leaves represents the digest of the block to which the packets belong. The size of a block needs to be chosen carefully to ensure that it does not introduce delay to collect all packets in it. If the tree is a $d$-ary tree, the height of the tree will be $\log _{d} \frac{F}{l}$, where $F$ is the size of the media file. The number of extra digests required to verify each block depends on the height of the tree. TFDP requires $(d-1)\left\lceil\log _{d} \frac{F}{N_{\min } l}\right\rceil$ digests to verify $N_{\text {min }}$ blocks. It can be easily shown that the number of extra digests to verify $N_{\text {min }}$ blocks is minimized when $d=2$.

\section{Comparison and Evaluation}

In [8], the authors show that SAIDA performs better than both EMSS [11] and Tree Chaining [16] in tolerating bursty packet losses. Therefore, we only compare TFDP with SAIDA and Tree Chaining. We compare the three protocols in terms of communication and computation overhead. The communication overhead is the extra bytes per packet client $P_{0}$ needs to receive from the suppliers and $S_{0}$ for data integrity verification. The computation overhead at $P_{0}$ is due to hash computation, signature verification, and FEC decoding.

Communication overhead. Tree Chaining requires $P_{0}$ to obtain the public key (usually 128 bytes long) of the server to verify the signature. $P_{0}$ needs to receive $l \log l$ digests for each block, where $l$ is the size of a block in number of packets. Each packet carries one 1,024-bit signature. Thus, for each block, $P_{0}$ needs to receive $20 l \log l+128 l$ bytes. TFDP requires only one digest ( 20 bytes) from the server. However, it needs $1+\frac{1}{N_{\min }} \log \left(\frac{M}{N_{\min }}\right)$ extra digests for each block. The digest of each block is encoded using FEC. We define $\alpha$, the overhead factor of FEC, as:

$$
\alpha=\frac{\text { total packets sent per block }}{\text { packets required to reconstruct the block }} \text {. }
$$

Thus, the total communication overhead of TFDP is $20(l \alpha+$ $\left.1+\frac{1}{N_{\min }} \log \left(\frac{M}{N_{\min }}\right)\right)$ bytes per block. SAIDA requires one signature per block, and it uses FEC. Thus, it incurs $(20 l+128) \alpha$ bytes of overhead for each block. Table 1 summarizes the comparison results.

We demonstrate the communication overhead of the three protocols using the trace of The Matrix movie. Fig. 2a shows that the communication overhead of SAIDA and TFDP is both low, because FEC is used to encode digests and signatures. On the other hand, Tree Chaining does not use FEC and incurs much higher communication overhead (208 bytes per packet, for $l=16$, not shown in Fig. 2a). TFDP incurs less overhead than SAIDA and Tree Chaining because TFDP verifies digests of every $N_{\min }$ data blocks as a group, which reduces the height of the Merkle tree from $\log M$ to $\log \frac{M}{N_{\min }}$. The difference in communication overhead between TFDP and SAIDA narrows when the block size gets larger. However, a large block size can cause delay during a streaming session because the client would need all packets in a block before it can reconstruct the block.

Computation overhead. In Tree Chaining, $M$ subtrees are created for $M$ blocks and each tree requires $2 l-1$ hash computation. SAIDA needs to decode digests for each packet and verify one signature for each block. Thus, the computation 


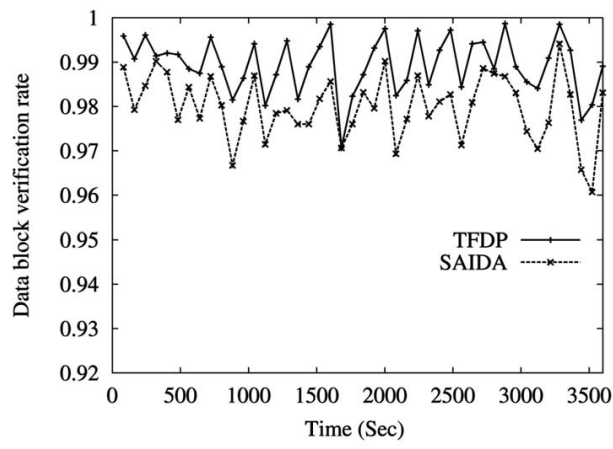

Fig. 3. Data block verification rate of SAIDA and TFDP (simulations).

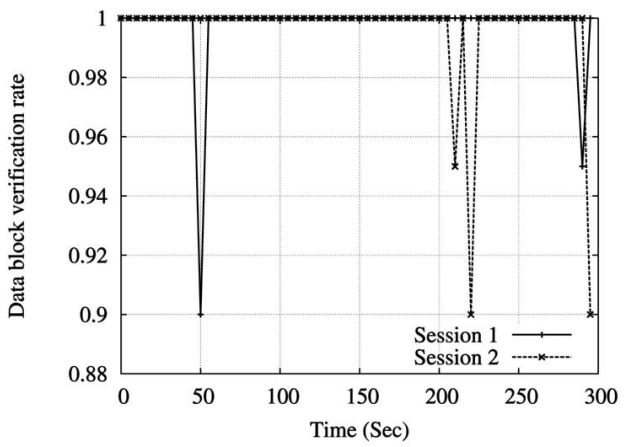

Fig. 4. Data block verification rate of TFDP (PlanetLab-based experiments).

overhead of SAIDA is due to $M(l+1)$ digests and $M$ signatures. TFDP needs to compute extra digests for every $N_{\min }$ blocks. The number of extra digests is $\mathrm{M} / N_{\min }\left[\left(N_{\min }-\right.\right.$ $\left.1)+\log \left(M / N_{\text {min }}\right)\right]$ and every block is verified during the streaming session. Thus, the total client-side computation overhead of TFDP is $M l+M / N_{\min }\left[\left(N_{\min }-1\right)+\log \left(M / N_{\min }\right)\right]$.

We use openSSL crypto library to calculate SHA-1 hash, RSA signature, and RSA verification. Cauchy-based Reed-Solomon code is used to encode digests. In Fig. 2b, we compare the computation overhead of the protocols using The Matrix movie. The computation overhead of Tree Chaining can be reduced by caching digests carried by previous packets. The cached digests are used to verify upcoming packets of a block. SAIDA incurs higher computation overhead than TFDP because SAIDA has to verify the signature of every block, which is more computation-intensive than verifying digests.

\section{Streaming Experiment Results}

We have conducted both streaming simulations and real-world experiments. In our simulations, one client requests and receives streaming media from five suppliers. Like in SAIDA, we use the two-state Markov loss model to introduce bursty packet losses. The parameters of the Markov loss model are $\operatorname{Pr}\{$ no loss $\}=0.95$ and $\operatorname{Pr}\{$ loss $\}=0.05$. The model characterizes the loss of every underlying network link connecting the five suppliers and the client. We define data block verification rate as the fraction of data blocks that can be verified during a certain time interval.

The simulation results of SAIDA and TFDP are shown in Fig. 3. In both protocols, the digests and signatures are FEC-encoded to tolerate 37.5 percent packet loss rate. We observe that, due to bursty packet losses, some blocks cannot be verified. The reason why TFDP performs better is that TFDP incurs slightly less communication overhead than SAIDA, which requires RSA signature for each block.
We have also developed a distributed media streaming system called PROMISE [3]. The system monitors network dynamics, quality of connections from multiple suppliers to a receiver, as well as supplier availability, to maintain full media playback quality on the client side. Particularly, the set of suppliers in a streaming session may change dynamically, so that the fluctuation of network and supplier conditions will not affect the client-side aggregated media streaming rate. TFDP can be integrated into PROMISE. We evaluate TFDP by conducting experiments in the wide-area PlanetLab testbed (www.planet-lab.org).

Fig. 4 shows the results from two streaming sessions using TFDP in PlanetLab. Both sessions can tolerate up to 20 percent packet loss due to FEC. In Session 1, TFDP is able to verify almost all the blocks during the first 300 seconds of the session. At the 50th second, the network loss rate goes up to 40 percent and the block verification rate temporarily drops to 0.9 . Session 2 experiences a few more glitches than Session 1. Still, in both sessions, the overall block verification rate achieved by TFDP remains high. The main reason is that PROMISE has dynamic supplier switching capability. The suppliers in a streaming session can be dynamically replaced when significant packet loss is experienced, in order to avoid the congested network links. Our experiments show that TFDP works well in the event of a dynamic supplier switch.

\section{Conclusion}

We study the problem of data integrity verification in distributed media streaming sessions. We propose a simple and efficient Treebased Forward Digest Protocol (TFDP) as our solution. TFDP incurs low communication and computation overhead, compared with existing data integrity verification protocols. More importantly, TFDP relieves the source server from supplying the digest of every data block in a streaming session. Instead, this load is distributed among the multiple supplying clients serving in the streaming session, enabling data integrity verification even if the suppliers are not trustworthy. Both simulation and Internet experiments demonstrate the effectiveness and practicality of TFDP.

\section{ACKNOWLEDGMENTS}

This work is supported in part by NSF-ITR 0085879, NSF-ANI 0219110, NSF-IIS 0209059, NSF-EIA 9903545, NSF-ISS 0219560, and CERIAS. This work was partially done when Ahsan Habib was at Purdue University.

\section{REFERENCES}

[1] D. Boneh, G. Durfee, and M. Franklin, "Lower Bounds for Multicast Message Authentication," Proc. Eurocrypt Conf., pp. 437-452, 2001.

[2] R. Gennaro and P. Rohatgi, "How to Sign Digital Streams," technical report, IBM T.J. Watson Research Center, 1997.

[3] M. Hefeeda, A. Habib, B. Botev, D. Xu, and B. Bhargava, "PROMISE: Peerto-Peer Media Streaming Using CollectCast," Proc. ACM Multimedia Conf., Nov. 2003.

[4] M.N. Krohn, M.J. Freedman, and D. Mazieres, “On-the-Fly Verification of Rateless Erasure Codes for Efficient Content Distribution," Proc. IEEE Symp. Security and Privacy, May 2004.

[5] L. Lamport, "Constructing Digital Signatures from a One-Way Function," Technical Report SRI-CSL-98, SRI Int'l Computer Science Laboratory, Oct. 1979.

[6] D.A. McGrew and A.T. Sherman, "Key Establishment in Large Dynamic Groups Using One-Way Function Trees," IEEE Trans. Software Eng., vol. 29, pp. 444-458, May 2003.

[7] R.C. Merkle, "A Certified Digital Signature," Proc. Conf. Advances in Cryptology, pp. 218-238, 1989.

[8] J.M. Park, E. Chong, and H. Siegel, "Efficient Multicast Packet Authentication Using Signature Amortization," Proc. IEEE Symp. Security and Privacy, May 2002

[9] A. Perrig, "The BiBa One-Time Signature and Broadcast Authentication Protocol," Proc. ACM Conf. Computer and Comm. Security, pp. 28-37, Nov. 2001. 
[10] A. Perrig, R. Canetti, D. Song, and D. Tygar, "Efficient and Secure Source Authentication for Multicast," Proc. Network and Distributed System Security Symp., Feb. 2001.

[11] A. Perrig, R. Canetti, J.D. Tygar, and D.X. Song, "Efficient Authentication and Signing of Multicast Streams over Lossy Channels," Proc. IEEE Symp. Security and Privacy, pp. 56-73, Nov. 2000.

[12] S. Rafaeli and D. Hutchison, "A Survey of Key Management for Secure Group Communication," ACM Computing Survey, vol. 35, no. 3, pp. 309-329, 2003.

[13] L. Reyzin and N. Reyzin, "Better than BiBa: Short One-Time Signatures with Fast Signing and Verifying," Proc. Seventh Australian Conf. Security and Information Privacy, Sept. 2002.

[14] R.L. Rivest, A. Shamir, and L.M. Adleman, "A Method for Obtaining Digital Signature and Public Key Cryptosystems," Comm. ACM, pp. 120126, Feb. 1978

[15] P. Rohatgi, "A Compact and Fast Hybrid Signature Scheme for Multicast Packet," Proc. ACM Conf. Computer and Comm. Security, pp. 93-100, Nov. 1999.

[16] C. Wong and S. Lam, "Digital Signatures for Flows and Multicasts," IEEE/ ACM Trans. Networking, vol. 7, no. 4, pp. 502-513, Aug. 1999.

$\triangleright$ For more information on this or any other computing topic, please visit our Digital Library at www.computer.org/publications/dlib. 